A UTHOR'S ABSTRACT OF THIS PAPLK IASUED

HY THE HIBL,IOGRAPHIC SERVICE, DECEMBER 8

\title{
STAINING OF ADULT CARTILAGE BY LUNDWALL'S METHODS
}

\author{
J. MOREIRA DA ROCHA
}

Fron Laboratories of Anatomy and Histology da Faculdade de Medicina $e$ Cirurgia de São Paulo (Brazil): Director, Dr. A. Bovero

In the Anatomical Section of the Primeiro Congresso Medico Paulista (December 5, 1916), I had the opportunity to present a series of excellent preparations from various organs of the body possessing cartilaginous skeleton, which were obtained by methods based upon the chromatic electivity of the cartilage and the clearing of the preparations.

In the same month of December, G. Noback (Anatomical Record, vol 11 , no. 5,1916 ), called attention to the excellent results which may be obtained in the embryonal cartilage, by the method proposed by Van Wijhe, which according to the author is very little known.

As we employed in the execution of our preparations of the adult cartilaginous skeleton, only the methods proposed by Lundwall (Anat. Anz., vol. 25, 1904; vol. 27, 1905; vol. 40, 1912) for the study of embryonal cartilage, we think that, specially after the appearance of Noback's note, it will be timely to give some considerations to this subject.

In 1914, Professor Bovero had the occasion to present to the Sociedade de Medicina e Cirurgia de Sao Paulo, a great number of preparations of the eartilage of the plica semilunaris oculi (third eyelid) of man and other mammals, obtained with the methylgreen staining method proposed by Lundwall ('05) for the embryonal cartilage, having at that time called attention to the fact that the preparations were not only elegant and demonstrable but also permanent.

Such preparations are unstable only when prepared of embryonal cartilage, it being noticed that their degree of stability is in the direct ratio of the degree of maturity of the cartilaginous tissue.

Following such steps in the laboratory, directed by Professor Bovero, we undertook to prepare a series of cartilaginous skeletons of the larynx, trachea and bronchi, of man and other vertebrate animals and of the cartilages of the nasal alae and septum; of the fibrous cartilage of the temporo-mandibular and sterno-clavicular joints; of the semilunar cartilages of the knee and of the calcaneous-astragalus-scaphoid joints; and of the cartilage of the auricle, all from man, but from different races and ages.

Some of the preparations were obtained by the methods proposed by Lundwall for the staining of cartilage alone and others were obtained by such modifications of the method as experience showed us to be of advantage. 
The first methor proposed by Lundwall, very well known nowa-days, which may be found in almost every text-book of histological technique (Ehrlich, Mosse and Krause, '10; Carazzi and Levi,' 11, et cetera), as also in some special memories (Bruni, '08), is based specially upon the staining with a 1 to 1000 solution of methylgreen in 70 per cent alcohol (lately the methylgreen has been substituted by toluidinblue) to which has been added a 7 to 1000 solution of acetic acid, with subsequent washing in 95 per cent alcohol until the preparation does not lose any more stain; dehydration by absolute alcohol; clearing by benzol changed many times; and finally conservation in a mixture of benzol, carbon sulphide and essence of peppermint. The changes made by Professor Bovero in this method, does not alter in any way the fundamental features of the method as devised by Lundwall, but they are to a certain point an incorporation in Lundwall's methods of analogous methods proposed by Bakay and by Van Wijhe.

We observed many times the fact, that the methylgreen obtained from different sources or even that obtained from the same source but at different times, does not react the same in the presence of the ground substance of the cartilage, specially in regard to the electivity of the staining and also as regards its stability. The causes of such variations in the action of the methylgreen, may be found in the fact that it is not a single stable compound, but a mixture of staining substances, and in fact that the different kinds of methylgreen employed by us, and with which we had negative or unsatisfactory results, had suffered some chemical decomposition.

Having always in mind the idea of the basophilic character of the ground substance of the cartilage, we used, besides the methylgreen, other substances such as toluidin blue, methylenblue, methylviolet and safranine. In some instances, we substituted the acetic acid by hydrochloric acid. The duration of the staining was largely modified as was also the temperature at which it took place, this, according to the size of the preparations and according to the varieties of cartilage that we tried to show. We used the benzol in very much less quantity than that advised by Lundwall, not only as a matter of economy but also because it was quite difficult to obtain this reagent here of the necessary purity. Thus, three, four or even more preparations were passed successively through the same amount of benzol. For this reason, it was necessary to leave the preparations immersed for a longer time in the benzol, to compensate for the less frequent change of the reagent.

We may say, as a general rule, that no matter which staining substance is used, its electivity for staining and its stability are in the direct ratio, as we have already said, not only to the maturity of the amorphous ground substance of cartilage, but also, what may be readily understood, to the quantity of the ground substance.

Therefore, we may establish a priori, that not only the adult, cartilage is stainable with greater chromatic affinity and also with greater stability than the embryonal cartilage, but that among the different 
varieties of adult cartilages, the hyaline variety will take the staining with greater electivity and with greater stability than the elastic and fibrous varieties, and this is readily verified in practice.

What we have endeavored to explain may be readily demonstrated by the simple test of the way in which the cartilage of the larynx and trachea behaves as against that of the knee and of the auricle.

Bearing closely to what we have said, we may add that it is necessary to watch with greater attention the decoloration of the elastic and fibrous cartilages, whereas it is not necessary to use such care as regards the hyaline cartilage.

Another advice may be given in regard to the embryonal and foetal cartilages, that is, that the conditions being the same, the staining with methylenblue or methylviolet, or with the toluidin blue is much more stable than with the methylgreen.

By the examination of our preparations we may conclude that such methods to demonstrate the cartilaginous tissue, always give constant and sure results and explain the possibility of a coming revision of full chapters of Anatomy dealing with the morphology of certain and determined cartilaginous organs, which were previously studied by the dissecting and macerating methods, followed by the desiccation of the cartilaginous pieces.

Our assertion seems to be perfectly justifiable in as much as none of these methods are as satisfactory to the one in which the cartilage is stained.

The revision of the morphology of the cartilaginous organs may give very good results, not only in regard to the larger hyaline cartilages (larynx, trachea, bronchi, nasal septum, et cetera,) but also as regards the study of the extension and topography of the amorphous ground substance in the fibrous cartilages, for example. We believe as regards this point of view that with the methods employed by us, combined with the methods for histologic research, interesting and new results may be obtained. Certainly, the free use of the methods of staining of the cartilaginous pieces in toto and their demonstration through the soft tissues promises to give many good results in any systematic studies which may be undertaken. On some of these, we expect to contribute very soon with personal and especial researches which we will develop during this year. 